\title{
Our experience with deceased organ donor maintenance
}

\author{
Meena Kumar, Prakash Shendge, Vineet Kumar, Vatsala Trivedi, Jagruti Waghela, Dilip Rajpal
}

Deceased organ donors in an intensive care unit (ICU) are the richest source of organs for transplantation. Careful donor maintenance plays a vital role in the successful functioning of the organ in the recipient. Aims: Early identification of brain stem death (BSD) in the ICU, problems and management in donor maintenance till retrieval are the main objectives. Materials and Methods: BSD was identified in a level I trauma center over a period of eight years (1996-2004) using UK code. After screening for fitness, they were maintained to achieve normothermia, systolic BP $>90 \mathrm{~mm} \mathrm{Hg}$, CVP $8-10 \mathrm{~cm}$ water, urine output $>80 \mathrm{ml} / \mathrm{hour}$ and normal acid base balance. Results: 168 cases of BSD were maintained, 30 with identity unknown. Common transient complications noted were hypotension $(68 \%)$, hypokalemia $(62 \%)$, hypothermia $(12 \%)$, diabetes insipidus (70\%). Brain stem death was identified early and resuscitated to maintain normal tissue perfusion. 17 (12.3\%) consent for organ donation was obtained. Organs (24 kidneys and one liver) were retrieved from 12 donors. Four donors sustained cardiac arrest before retrieval. Conclusion: Early recognition of brain stem death and prompty correction of hemodyanamic instability is the key to deceased donor maintenance. Optimal care of potential donor translates to care of multiple recipients.
\end{abstract}

Key words: Brain Stem Death (BSD), deceased organ donor, maintenance

\section{Introduction}

Brain stem dead (BSD) cases are the single largest source of organs for transplantation. The various organs that can be donated are heart, lungs, kidneys, liver, pancreas and intestine. Deceased organ donation is a complex process with discrete interconnected steps like donor identification, screening, maintenance, consent organ retrieval and organ allocation. ${ }^{[1]}$ After initial identification of BSD in the intensive care unit (ICU), the deceased donor needs to be screened for fitness for organ donation. Once a fit potential donor is identified
From:

Department of Surgery, Lokmanya Tilak Municipal General Hospital, Sion, Mumbai, *Sir H N Hospital, Mumbai, India

Correspondence to:

Dr Meena Kumar, Department of Surgery, Lokmanya Tilak Municipal General Hospital, Sion, Mumbai, India. E-mail: meenavkumar@ rediffmail.com the case has to be maintained in optimal conditions till organs are retrieved after consent from next of kin. It is necessary that the organs are well perfused with oxygenated blood till organs are retrieved. Maintaining these donors is a complicated challenge. The key to deceased donor maintenance is to identify brain stem death early and manage early the common complications like hypotension, arrythmias, electrolyte imbalance, alteration in pulmonary functions, diabetes insipidus, hypothermia, hyperglycemia and coagulopathy. ${ }^{[2-4]}$ Optimal care of potential donor results in successful outcome in recipients. ${ }^{[2,5,6]}$

\section{Materials and Methods}

Brain stem dead cases were identified in the ICU of a municipal Level I trauma center over eight years from 1996-2004. 


\section{Inclusion criteria}

Age 3-65 years,

Patient in ICU in coma,

Known cause for coma,

Patient on ventilator.

\section{Exclusion criteria}

Metabolic / endocrine coma,

Long standing hypertension, diabetes mellietus, cardiovascular disease,

Primary hypothermia $<35^{\circ} \mathrm{C}$,

Malignancy.

All patients with GCS 5 and less on admission or had deteriorated to GCS 5 were closely monitored for development of brain stem death after failure of aggressive management to reverse coma. ${ }^{[4]}$ Brain stem death was identified clinically using UK code which involved satisfying preconditions like patient in an ICU on a ventilator with known cause of coma. ${ }^{[3,5,7]}$ All drug induced, metabolic causes of coma were excluded. Brain stem death was confirmed by absent brain stem reflexes and a strict apnoea test. Once brain stem death was confirmed the potential organ donors were screened for fitness to donate organs according to Maharashtra State appropriate authority guidelines. ${ }^{[7]}$ Confirmation of brain stem death was done twice within a gap of at least six hours.

All patients had a central line, systolic blood pressure was maintained $>90 \mathrm{~mm}$ mercury, CVP $8-12 \mathrm{~cm}$ water, urine output maintained between $80-100 \mathrm{ml}$ per hour. IV fluids used were Bolus Colloid, Ringer Lactate, Dextrose Normal Saline. Injection Mannitol which was being used for decreasing cerebral edema was withdrawn once BSD was identified or in case of hypernatraemia. All patients were on ventilator with $\mathrm{PaO} 2$ more than 100 $\mathrm{mm}$ mercury, PCo2 of $35 \mathrm{~mm} \mathrm{Hg}$ and $\mathrm{pH}$ more than $7.2 .^{[3,7]}$ The deceased donor with unknown identity were maintained hoping close relatives would arrive.

\section{Investigations}

CBC, BUN, S.Cr, LFT, blood sugar, blood group, viral markers, urine-routine, microscopy, X-ray chest, ECG, USG abdomen

$A B G$ and serum electrolytes were monitored 8 hourly

\section{Management $t^{[5,6,8-10]}$}

i. IV fluids - previous hours urine output plus $25 \mathrm{cc}$, maintaining CVP, BP, serum sodium less than 150.

ii. Hypotension was managed with Bolus Colloid, Dopamine 3-5 microgms $/ \mathrm{kg} / \mathrm{min}$, dobutamine 5-10 micrograms $/ \mathrm{kg} / \mathrm{min}$, adrenaline 1 microgram $/ \mathrm{kg} /$ $\min$.

iii. Bradycardia / arrhythmia was managed by correction of electrolyte imbalance, acid base imbalance, oxygenation, treatment of hypothermia, inotropes and in a few cases trans venous pacing.

iv. Body temperature was maintained more than 35 degree centigrade with warming blankets, $60 \mathrm{~W}$ overhead bulb and if necessary warm gastric and rectal washes.

v. Nutrition was maintained with enteral feeds.

vi. Broad-spectrum antibiotics was given as per ICU protocol.

vii. General ICU principles for patient care were followed.

viii. Intense close monitoring of patients were done.

ix. Any cardiac arrest with prolonged hypotension of less than $70 \mathrm{~mm} \mathrm{Hg}$ for more than 30 min was considered as relative contraindication.

$x$. Professor (Department of General Surgery) is incharge of Trauma ICU and manages in co-ordination with anesthesiologists. The certification of BSD was done by the authorized committee. The transplant team was different from the team managing the ICU. Counseling was done with the help of medical social workers.

The doctors in ICU were trained for early detection and management of BSD donors.

\section{Results}

On maintaining 168 BSD cases in a level 1 trauma centre the observations and result are as shown in Tables 1-11.

\section{Discussion}

Our hospital has a 14-bedded trauma ICU with annual admission of 2800 cases of intensive trauma. 168 cases of brain death were maintained from 1996 - 2004. All GCS 5 and less patients either on admission or deterioration to GCS 5 even after aggressive management were closely 


\section{Table 1: Distribution of cases}

\begin{tabular}{lc}
\hline Cases & Number $(\%)$ \\
BSD maintained & 168 \\
Unknown & $30(17.9)$ \\
Males & $148(88)$ \\
Females & $20(12)$ \\
\hline
\end{tabular}

\section{Table 2: Age distribution}

\begin{tabular}{lcc}
\hline Age (years) & Number & $\%$ \\
$3-14$ & 8 & 4.7 \\
$15-35$ & 113 & 67.5 \\
$36-55$ & 37 & 22 \\
Up to 65 & 10 & 5.8 \\
\hline
\end{tabular}

Table 3: Mode of injury

\begin{tabular}{lc}
\hline Mode & Number (\%) \\
Vehicular & $63(37.5)$ \\
Railway & $47(28)$ \\
Fall & $45(26.8)$ \\
Assault & $08(4.8)$ \\
Occupational & $05(2.9)$ \\
\hline
\end{tabular}

\section{Table 4: GCS on admission}

\begin{tabular}{lc}
\hline GCS & Number $(\%)$ \\
$3-5$ & $144(85.7)$ \\
$6-9$ & $18(10.7)$ \\
$10-12$ & $06(3.6)$ \\
\hline
\end{tabular}

\section{Table 5: CT findings}

\begin{tabular}{lcc}
\hline CT findings & Number & $\%$ \\
Extra dural hematoma & 34 & 20.2 \\
Sub dural hematoma & 41 & 24.4 \\
Sub arachanoid H'rhage & 41 & 24.4 \\
Contusion & 70 & 41.7 \\
Edema & 79 & 47 \\
Compound fracture & 26 & 15.5 \\
Simple fracture & 24 & 14.3 \\
Base skull fracture & 10 & 5.9 \\
Shear injury & 14 & 8.3 \\
\hline
\end{tabular}

\section{Table 6: Treatment offered}

\begin{tabular}{lc}
\hline Treatment & Number (\%) \\
Operated & $75(44.5)$ \\
Non operated & $93(55.5)$ \\
\hline
\end{tabular}

Table 7: Time to declaration of brain stem death after admission

\begin{tabular}{lc}
\hline Day of declaration of brain stem death & Number $(\%)$ \\
$>12$ hours $<2$ days & $130(77)$ \\
$3^{\text {rd }}$ day & $20(12)$ \\
$>4$ days & $18(11)$ \\
\hline
\end{tabular}

observed for development of brain stem death. Early identification of brain stem death can help in tackling hemodyanamic instability at the earliest so that we have optimally functioning donor organs. The sex ratio of potential donor was 7 Males: 1 Female and the average age group was $15-35$ years (67\%) [Table 1 and 2]. The
Table 8: Common transient complications

\begin{tabular}{lc}
\hline Complication type & Number (\%) \\
Hypotension & $114(68)$ \\
Diabetes insipidus & $117(70)$ \\
Poikilothermia & $20(12)$ \\
Hypokalemia & $103(61.3)$ \\
Arrhythmia & $27(16.1)$ \\
Cardiac arrest needing CPR & $29(17.3)$ \\
Acidosis & $12(7.1)$
\end{tabular}

Table 9: lonotropic support

\begin{tabular}{lcc}
\hline Drugs & Number & $\%$ \\
Dopamine & 165 & 98 \\
Dobutamine & 20 & 12.2 \\
Adrenaline & 32 & 19 \\
\hline
\end{tabular}

Table 10: Duration of maintenance of brain stem death cases

\begin{tabular}{lc}
\hline Time in hours & Number $(\%)$ \\
$6-12$ & $05(3)$ \\
24 & $145(86.3)$ \\
48 & $17(10.1)$ \\
72 & $01(0.6)$ \\
\hline
\end{tabular}

\begin{tabular}{lc}
\hline Table 11: Donor and analysis & \\
\hline Outcome & Number \\
Maintained deceased donors & 168 \\
Unknown & 30 \\
Counseled for donation & 138 \\
Consent & $17(12.3 \%)$ \\
Organ retrieved & 12 donors $=24$ kidneys +1 liver \\
Trans venous pacing & 06 \\
Longest maintained & 72 hours \\
\hline
\end{tabular}

common modes of injury were vehicular $(37.6 \%)$, railways (28.2\%), fall (27\%) [Table 3]. This was as per the trauma admission pattern of the hospital. The GCS on admission was between 3-5 (85.8\%) [Table 4]. The predominant CT findings were cerebral edema (47\%), contusion (41.8\%), SAH (24.7\%), SDH (24.7\%) [Table 5]. 55.5\% cases were managed nonoperatively and $44.5 \%$ cases were operated on admission [Table 6]. We followed the UK criteria for identification of BSD and potential deceased donors were maintained after screening them for fitness for organ donation. The diagnosis of brain stem death was done within 2 days of admission in $77 \%$ of cases, $3^{\text {rd }}$ day in $12 \%$ and after 4 days in $11 \%$ of cases [Table 7]. GCS 3 cases on admission were intubated and put on ventilator without any muscle relaxant. All trauma patients with GCS 5-8 were on controlled mode ventilation with Vecuronium as muscle relaxant, which was repeated every 15-20 minutes as they came out of its effect. It was critical to identify the transition from life to brain stem death. Cases not requiring Vecuronium for more than 10-12 hours were suspected and tested for brain stem 
reflexes. Nerve stimulation studies were not conducted routinely. One of the other early signs a staff nurse could pick up in an ICU was the loss of gag reflex and high urine output. The early detection of brain stem death required an attitudinal change amongst doctors in ICU as one had to switch over to high intensive monitoring of GCS 5 and less cases with extensive head injury who otherwise had poor outcome. Each case was given a minimum of 12 hours of aggressive anti-coma management before BSD declaration. These brain stem dead cases went through swinging changes in hemodyanamics like hypotension $68 \%$, diabetes insipidus $70 \%$, hypothermia $12 \%$, hypokalemia $62 \%$ [Table 8]. Close monitoring and rapid correction of complication was done to minimize fluctuations in tissue perfusion. Dopamine, adrenaline and dobutamine Table 9 were the inotropes used.

Brain stem death was identified, screened and certified and consent from next of kin taken. The heart beating deceased donor was then shifted to the operation theatre for organ retrieval. The average time gap between certification and consent varied from few minutes to 24 hours $(86.2 \%)$. This was due to delay in obtaining consent [Table 10]. One deceased donor was maintained for 72 hours as close relatives were not available for consent. The pressure on the team maintaining the deceased donors after obtaining consent till organ retrieval is very high. In the early part of our experience, in spite of a positive consent, we lost 4 donors as they arrested before organ retrieval. After the initial setback in case of instability Trans venous pacing was done in six cases especially when there was likelihood of consent for donation. One of the factors on which primary allograft function depends on is a well-maintained donor.

Our hospital being a municipal public hospital mainly looks after people from low socioeconomic strata. Of the 138 potential donors counseled, consent was obtained in $17[12.3 \%]$ but organ retrieval was done from 12 donors as four donors arrested before retrieval and one was $\mathrm{HbsAg}+$ with no corresponding recipient available in the city. Most of the retrievals were done from four to eight hours after consent. 24 kidneys and one liver were retrieved and successfully transplanted [Table 11]. The critical condition and prognosis of patients in trauma ICU was explained to the relatives by the senior surgeons in charge. The brain stem death declaration to relatives was also done by the seniors. Once the relatives were convinced about death they were approached for organ donation by the counselor. It was observed that it took some time for the relatives to accept that their young trauma patient was seriously injured and brain dead. They went through phases of shock and anger. Acceptance of brain stem death in a still warm patient was difficult. None of them had heard earlier about organ donation. The poor consent rate is attributed to lack of awareness about organ donation in general, inability to take decision regarding organ donation. Those who gave consent were from educated middle class families, were not aware of organ donation but were willing to donate. Some of these patient's relatives, friends/family members had died of kidney failure. Apathy and hesitancy amongst health care workers and nonavailability of regular counselor in our hospital also contributed to the poor response rate. Maintaining the potential donors in a public hospital is high manpower, resource and cost intensive.

\section{Conclusion}

Well-maintained BSD cases are the rich sources of transplantable organs. As BSD cases undergo swinging hemodyanamic instability, they need to be picked up early and maintained with close monitoring and intensive care. On maintaining 168 BSD cases within constraints of resources and manpower in trauma ICU of a public busy trauma center resulted in consent of $12.3 \%$ and successful transplantation. Optimal pre and intra op management of deceased donors consists of correction of fluid and electrolyte imbalance, treatment of diabetes insipidus, use of inotropes, treatment of hypothermia, use of ventilator and enteral nutrition so that tissue perfusion, oxygenation and normothermia is maintained. Management of brain stem dead cases is a complicated challenge, which requires a team of dedicated and motivated health care workers.

\section{Acknowledgement}

We acknowledge the deceased donors and their relatives, Dr. Magore, the Head of Department of Surgery and the Dean of L T M M C and General hospital.

\section{References}

1. Pallis C. Brain Stem Death the evolution of a concept in Kidney Transplantation Principles and Practice. Morris PJ, editor. $3^{\text {rd }}$ ed. 1988. p. 123-50.

2. Marpshall VC, Jablenski P, Scott DF. Renal preservation in Kidney Transplantation. Morris PJ, editor. $3^{\text {rd }}$ ed. 1988. p. 151-82. 
3. Power DM, Van Hearden TV. The physiological changes associated with brain death - current concepts and implications for treatment of the brain dead organ donor. Anaesth Intensive Care 1995;23:26-36.

4. Nygaard CE, Townsend RN, Diamond DL. Organ donor management and organ outcome: A 6 year review from a level I trauma centre. J Trauma 1990;30:728-32.

5. Pallis C. ABC of brain stem death. The declaration of death. $\mathrm{Br}$ Med J 1983;286:39.

6. Razek T, Olthoff K, Reilly PM. Issues in potential organ donor management. Surg Clin North Am 2000;80:1021-32.

7. Guidelines/standards for cadaveric organ transplant by Appropriate
Authority and Director of Health Services of Maharashatra. 1999. p. 4-10.

8. Wood KE, Becker BN, McCartney JG, D'Alessandro AM, Coursin DB. Care of potential organ donor. N Engl J Med 2004;351:27309.

9. Jenkins DH, Reilly PM, Schwab CW. Improving approach to organ donation: Review. World J Surg 1999;23:644-9.

10. Soifer B, Gelb AW. The multiple organ donor: Identification and management. Ann Intern Med 1989;110:814-23.

Source of Support: Nil, Conflict of Interest: None declared 